safety of TACE combined with microwave ablation (MWA) versus TACE combined with cryoablation (CRA) in treating unresectable HCC.

Methods From January 2011 to December 2018, 108 patients diagnosed with unresectable HCC were divided into either the TACE-MWA group $(n=48)$ or TACE-CRA group $(n=60)$. Overall survival (OS) and time to progression (TTP) were compared between the two groups. Complications were observed. Kaplan-Meier survival curves were constructed and compared using the log-rank test.

Results Baseline characteristics of the two groups were basically balanced. The median OS was 20.9 months (95\% CI 14.3-27.6 months) in the TACE-MWA group and 13.0 months (95\% CI 8.8-17.1 months) in the TACE-CRA group $(\mathrm{P}=.096)$. The median TTP was 8.8 months (95\% CI 4.313.4 months) in the TACE-MWA group and 9.3 months (95\% CI 7.1-11.5 months) in the TACE-CRA group $(\mathrm{P}=.675)$ (figure 1). The overall incidence rate of ablation-related complications was lower in the TACE-MWA group than in the TACE-CRA group $(66.7 \%$ vs. $88.3 \%, \mathrm{P}=.006)$. Multivariate analysis showed that the presence of portal vein tumor thrombus (PVTT) and the maximum diameter of the intrahepatic tumor were significant prognostic factors for OS and TTP.

Conclusions The efficacy of TACE-MWA and TACE-CRA in the treatment of unresectable HCC was comparable. TACEMWA was more promising because of a lower complication rate, especially with regard to thrombocytopenia. Further prospective randomized controlled trials are required to validate our findings.

\section{IDDF2020-ABS-0064 THREE-DAY POSTOPERATIVE ANTIMICROBIAL PROPHYLAXIS CAN REDUCE THE INCIDENCE OF POSTOPERATIVE INFECTION IN PRIMARY HEPATOCELLULAR CARCINOMA: A MULTICENTER RETROSPECTIVE STUDY}

${ }^{1}$ Zebin Chen* ${ }^{1}$ Yuanqi Wang, ${ }^{2}$ Ruiming Liang, ${ }^{3}$ Jiaming Lai, ${ }^{1}$ Dongming Li, ${ }^{1}$ Shaogiang ${ }^{1}$ Kai Lei, ${ }^{2}$ Qian Zhou, ${ }^{1}$ Baogang Peng. ${ }^{1}$ Department of Liver Surgery, the First Affiliated Hospital, Sun Yat-sen University, China; ${ }^{2}$ Clinical Trials Unit, the First Affiliated Hospital, Sun Yat-sen University, China; ${ }^{3}$ Department of Pancreato-biliary Surgery, the First Affiliated Hospital, Sun Yat-sen University, China

\subsection{6/gutjpl-2020-IDDF.147}

Background Postoperative infection in primary hepatocellular carcinoma (HCC) may cause bad consequences, even affect overall survival. But the evidence of using postoperative antimicrobial prophylaxis (AMP) in HCC was not sufficient enough. We aimed to explore the relationship between postoperative AMP and infection after hepatectomy.

Methods We retrospectively collected 1648 HCC patients who underwent hepatectomy from three tertiary hospitals. The incidences of postoperative infection, including surgical site infection (SSI) and remote site infection (RI) were recorded and calculated. Univariable and multivariable Cox regression analyses were performed to explore risk factors of postoperative infection. Inverse probability of treatment weighting (IPTW) analysis was also performed to reduce the selection bias.

Results The overall infection rate was 9.7\% (160/1648), including $8.1 \%$ of SSI and $2.3 \%$ of RI. Multivariable analysis revealed that the duration of postoperative AMP was negatively related to the incidence of postoperative infection significantly (OR 1.39 , 95 per cent c.i. 1.28 to $1.52 ; P<0.01)$.
And 3-day regimen seemed to be the shortest duration of postoperative AMP to gain the lowest incidence of postoperative infection. In the subgroup analysis between patients received 0 -day and 3-day postoperative AMP, using postoperative AMP for 3 days was an independent protective factor of infection (OR 0.04, 95 per cent c.i. 0.01 to 0.29 ; $P<0.01$ ). IPTW analysis showed consistent results with those of previous analysis $(P=0.01)$.

Conclusions Postoperative AMP is necessary for HCC patients to prevent postoperative infection. Using antibiotics based on the experience of surgeons for 3 days after surgery might be proper.

(Figure 1)

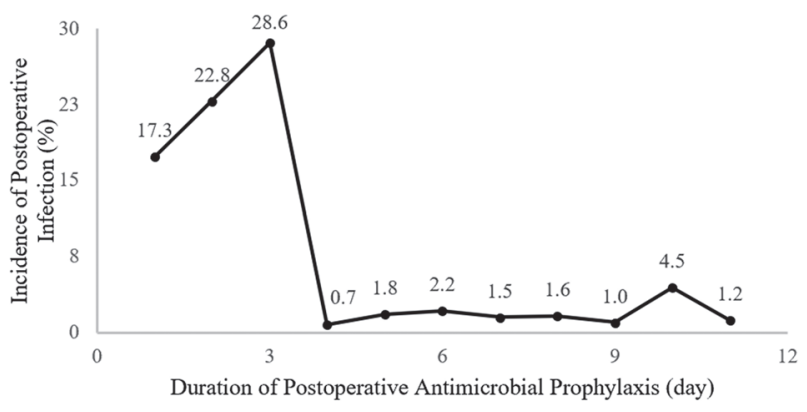

Abstract IDDF2020-ABS-0064 Figure 1 Duration of postoperative antimicrobial prophylaxis (day)

\section{IDDF2020-ABS-0065 SAFETY AND EFFICACY OF LAPAROSCOPIC MICROWAVE ABLATION AND PORTAL VEIN LIGATION FOR STAGED HEPATECTOMY (LAPS) IN PATIENTS WITH HBV-RELATED HEPATOCELLULAR CARCINOMA}

${ }^{1}$ Kai Lei*, 'Zebin Chen, ${ }^{2}$ Shuling Chen, ${ }^{3}$ Ruiming Liang, ${ }^{3}$ Zhenwei Peng, ${ }^{3}$ Sui Peng. ${ }^{1}$ Department of Liver Surgery, The First Affiliated Hospital of Sun Yat-sen University, China: ${ }^{2}$ Division of Interventional Ultrasound, The First Affiliated Hospital of Sun Yat-sen, China; ${ }^{3}$ Clinical Trials Unit, The First Affiliated Hospital of Sun Yat-sen University, China

\subsection{6/gutjnl-2020-IDDF.148}

Background Associating liver partition and portal vein ligation for staged hepatectomy (ALPPS) has high morbidity and mortality. In this study, the safety and efficacy of a modification of ALPPS (laparoscopic microwave ablation and portal vein ligation for staged hepatectomy, LAPS) were compared with the classic ALPPS in patients with HBV-related hepatocellular carcinoma (HCC).

Methods Patients who were diagnosed with HCC and were considered to have insufficient future liver remnant (FLR) were enrolled. In stage I, a microwave ablation (MWA) device was used to cauterise along the planned transection plane to form a coagulum avascular area. When the FLR reached above $40 \%$, hepatectomy was performed in stage II along the coagulum area established previously. After two stages, operative morbidity, mortality, increase in FLR, operative time and blood loss were evaluated.

Results Between April 2013 and September 2019, 7 patients with HBV-related HCC were treated with the LAPS procedure, and 14 patients were treated with the ALPPS procedure. No major complications (Clavien-Dindo IIIa) occurred after 1 stages of the LAPS group, while the ALPPS group were $21.4 \%(3 / 14)$. Completion rate of secondary surgery of the 
LAPS and the ALPPS was $85.7 \%(6 / 7)$ and $78.6 \%(11 / 14)$. The incidence of major complications was $36.4 \%(4 / 11)$ of the ALPPS group and $50.0 \%(3 / 6)$ of the LAPS group after the 2 stages operation. One patient died of the ALPPS group. Additionally, the median increase in FLR, median operative time and blood loss during the two stages of the LAPS were similar to those subjected to ALPPS.

Conclusions LAPS has a potential advantage in eliminating major complications of PHLF associated with classic ALPPS. LAPS may achieve the same effect of promoting significant growth of the FLR in patients with HBV-related HCC, albeit at the cost of longer interval time.

\section{IDDF2020-ABS-0069 PREDICTION OF MICROVASCULAR INVASION BEFORE SURGERY IN PATIENTS WITH HEPATOCELLULAR CARCINOMA: A NOMOGRAM MODEL BASED ON INFLAMMATORY MARKERS}

${ }^{1}$ Qianwen Zeng*, ${ }^{2}$ Han Xiao, ${ }^{3}$ Tingfan Wu, ${ }^{3}$ Xin Li, ${ }^{4}$ Sui Peng, ${ }^{5}$ Ming Kuang. 'Zhongshan School of Medicine, Sun Yat-sen University, China; ${ }^{2}$ Division of Interventional Ultrasound, The First Affiliated Hospital of Sun Yat-sen University, China; ${ }^{3}$ GE Healthcare, Beijing, China; ${ }^{4}$ Clinical Trials Unit, Department of Gastroenterology, The First Affiliated Hospital of Sun Yat-sen University, China; ${ }^{5}$ Department of Liver Surgery, Cancer Center, Precision Medicine Institute, The First Affiliated Hospital of Sun Yat-sen University, China

\subsection{6/gutjpl-2020-IDDF.149}

Background Microvascular invasion (MVI) remains a risk factor for tumor recurrence and metastasis in hepatocellular carcinoma (HCC). No effective and well-recognized method can detect MVI before surgery. Inflammatory markers reflect the immune environment and have been proven to be related to prognosis as well as the presence of MVI in HCC. We aimed to establish an MVI predictive model based on inflammatory markers.

Methods Data of 1058 cases of HCC patients treated in the First Affiliated Hospital of Sun Yat-sen University from November 2003 to December 2015 were collected. In a ratio of $7: 3$, patients were divided into the training group (740 cases) and the validating group (318 cases). Inflammatory factors related to MVI diagnosis in HCC patients were selected by LASSO regression analysis, and were then integrated into an 'Inflammatory Score'. A prognostic Nomogram model was established by combining the Inflammatory Score and the independent factors determined by multivariate logistic regression analysis. The consistency index (C-index) and the area under the curve (AUC) were used to evaluate the predictive efficacy of the model.

Results A total of 1058 HCC patients were included in this retrospective study, 430 of whom $(40.6 \%)$ were diagnosed with MVI. Sixteen inflammatory factors, including neutrophil, neutrophil to lymphocyte ratio, platelet to lymphocyte ratio, etc., were selected by LASSO regression analysis to establish an Inflammatory Score. Multivariate logistic regression analysis showed that Inflammatory Score $(\mathrm{OR}=2.186,97.5 \% \mathrm{CI}$ : 1.656-2.950), age (OR $=0.987,97.5 \% \mathrm{CI}: 0.973-1.000)$, alpha fetoprotein $(\mathrm{OR}=1.923,97.5 \%$ CI: 1.380-2.690), tumor size $(\mathrm{OR}=2.308,97.5 \% \mathrm{CI}$ : 1.656-3.220) were independent factors in the diagnosis of MVI in HCC patients. These four factors were then used to establish a Nomogram for MVI prediction. The C-index of the Nomogram prediction model was 0.72 . The AUC for the training and validating group were 0.720 and 0.721 , respectively.
Conclusions The Nomogram prediction model drawn in this study has a high prognostic value, which is capable of improving the diagnosis efficiency of MVI in HCC patients.

\section{IDDF2020-ABS-0078 IMMUNOSCORE CLASSIFICATION FROM HEPATOCELLULAR CARCINOMA HISTOPATHOLOGY IMAGES USING DEEP LEARNING: A PRELIMINARY STUDY}

${ }^{1}$ Qiaofeng Chen*, ${ }^{2}$ Yunquan Gu, ${ }^{2}$ Ruixuan Wang, ${ }^{3}$ Han Xiao, ${ }^{4}$ Sui Peng, ${ }^{5}$ Ming Kuang. ${ }^{1}$ Department of Gastroenterology, the First Affiliated Hospital, Sun Yat-sen University, China; ${ }^{2}$ School of Data and Computer Science, Sun Yat-sen University, China; ${ }^{3}$ Division of Interventional Ultrasound, the First Affiliated Hospital, Sun Yat-sen University, China; ${ }^{4}$ Clinical Trials Unit, the First Affiliated Hospital, Sun Yat-sen University, China; ${ }^{5}$ Department of Liver Surgery, the First Affiliated Hospital, Sun Yat-sen University, China

\subsection{6/gutjnl-2020-IDDF.150}

Background Immunotherapy is a recent advance for the treatment of hepatocellular carcinoma (HCC). Immunoscore assessment plays a critical role in precision immunotherapy and can predict prognosis in patients with HCC. This study aims to develop a deep-learning model to automated analyze histopathology images for classification of immunoscore (CD3 or CD8, 0-2 vs. 3-4) in HCC.

Methods We trained a patch-based deep convolutional neural network (Resnet-18) on whole-slide images to automatically classify immunoscore into $0-2$ or 3-4. The data were randomly split into a training and testing dataset. The performance was first estimated on the training dataset with ninefolded cross-validation and then further validated on the testing dataset. Cross-entropy was used as a model-optimized loss function and the accuracy as well as the area under the receiver operating characteristic curve (AUC) were calculated for the identification values. Heatmaps were also generated by our model to visualize the regions the most associated with the classification.

Results We included 28 images from a study cohort of 28 HCC patients for training (18 images) and testing (10 images) the model. After iterative training, an optimized architecture

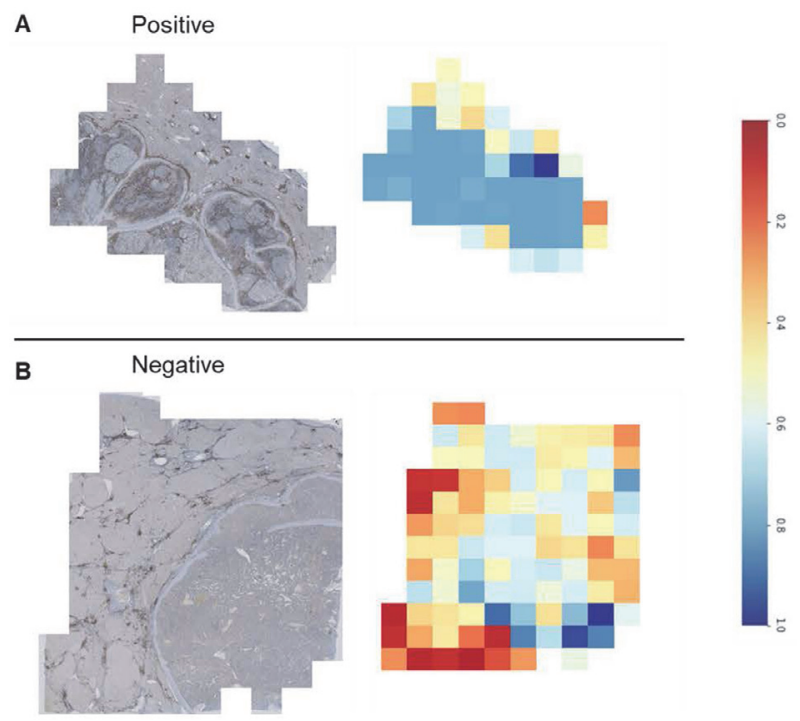

Abstract IDDF2020-ABS-0078 Figure 1 Heatmaps of patch-based tissue slides generated by the model in the testing dataset 